\title{
The Link between Cognitive Measures and ADLs and IADL Functioning in Mild Alzheimer's: What Has Gender Got to Do with It?
}

\author{
James R. Hall, ${ }^{1,2}$ Hoa T. Vo, ${ }^{1}$ Leigh A. Johnson, ${ }^{3}$ Robert C. Barber, ${ }^{2,4}$ and S. E. O’Bryant ${ }^{5,6}$ \\ ${ }^{1}$ Department of Psychiatry and Neuroscience, University of North Texas Health Science Center, \\ 3500 Camp Bowie Boulevard, Fort Worth, TX 76107, USA \\ ${ }^{2}$ Institute of Aging and Alzheimer's Disease Research, University of North Texas Health Science Center, Fort Worth, TX 76107, USA \\ ${ }^{3}$ Department of Family and Community Medicine, Texas Tech University Health Sciences Center, \\ and Laura W. Bush Institute for Women's Health, Lubbock, TX 79430, USA \\ ${ }^{4}$ Department of Pharmacology and Neuroscience, University of North Texas Health Science Center, Fort Worth, TX 76107, USA \\ ${ }^{5}$ F. Marie Hall Institute for Rural and Community Health, Texas Tech Health Sciences Center, Lubbock, TX 79430, USA \\ ${ }^{6}$ Department of Neurology, Texas Tech University Health Sciences Center, Lubbock, TX 79430, USA
}

Correspondence should be addressed to James R. Hall, james.hall@unthsc.edu

Received 15 December 2010; Revised 9 March 2011; Accepted 22 March 2011

Academic Editor: Francesco Panza

Copyright () 2011 James R. Hall et al. This is an open access article distributed under the Creative Commons Attribution License, which permits unrestricted use, distribution, and reproduction in any medium, provided the original work is properly cited.

Objectives. To investigate the link between neurocognitive measures and various aspects of daily living (ADL and IADL) in women and men with mild Alzheimer's disease (AD). Methods. Participants were 202 AD patients (91 male, 111 female) with CDR global scores of $\leq 1$. ADLs and IADLs ratings were obtained from caregivers. Cognitive domains were assessed with neuropsychological testing. Results. Memory and executive functioning were related to IADL scores. Executive functioning was linked to total ADL. Comparisons stratified on gender found attention predicted total ADL score in both men and women. Attention predicted bathing and eating ability in women only. Language predicted IADL functions in men (food preparation) and women (driving). Conclusions. Associations between ADLs/IADLs and memory, learning, executive functioning, and language suggest that even in patients with mild $\mathrm{AD}$, basic ADLs require complex cognitive processes. Gender differences in the domains of learning and memory area were found.

\section{Introduction}

Basic and instrumental activities of daily living are tasks required to function on a daily basis, and are often impacted by disease processes that reduce cognitive ability such as Alzheimer's disease (AD). Basic activities of daily living (ADLs) include core tasks of everyday life such as eating, dressing, grooming, and bathing while instrumental activities of daily living (IADLs) include more complicated, higher-level, tasks such as preparing meals, managing finances, shopping, doing housework, and using the telephone. Driving and medication management are other IADLs that are significantly disturbed in patients with $\mathrm{AD}[1]$. While both ADLs and IADLs are impacted by $\mathrm{AD}$, IADLs are the first to decline and the level of functional impairment is the core clinical distinction between $\mathrm{AD}$ and milder conditions such as mild cognitive impairment (MCI). Classification of MCI subtypes, suggest that MCI-amnestic type and MCI patients with multipledomain impairments are more predictive of later conversion to dementia and are more impaired functionally compared to MCI of nonamnestic and single domain subtype [2].

Patients with $\mathrm{AD}$ experience a gradual loss in the abilities to live independently due to impairments in cognitive and memory functioning $[1,3]$ and, as the disease progresses, the ability to carry out these essential activities eventually disappears [4]. Functional impairments in $\mathrm{AD}$ place the greatest burden on both caregivers and the economy [5]. Although there is not a large body of research on the relationship between neuropsychological measures and functional activities, several studies suggest that neuropsychological test 
TABle 1: Demographic characteristics of the sample.

\begin{tabular}{lccc}
\hline & Male $(n=99)$ & Female $(n=111)$ & $P$ \\
\hline MMSE $(M$, SD) & $21.53(4.59)$ & $20.95(4.47)$ & .364 \\
Age & $74.36(8.21)$ & $76.95(7.74)$ & .022 \\
Education & $15.42(2.87)$ & $13.74(3.00)$ & $<.0001$ \\
CDR total & $5.45(2.87)$ & $5.93(3.36)$ & .283 \\
CDR global & $.91(.45)$ & $1.03(.57)$ & .089 \\
GDS total & $4.59(4.14)$ & $5.49(5.76)$ & .233 \\
Estimated IQ & $108.22(13.88)$ & $107.70(16.58)$ & .819 \\
\hline
\end{tabular}

M: Mean, SD: standard deviation, IQ: intelligence quotient, CDR: clinical rating scale.

performance is predictive of complex ADLs and IADLs in elderly neuropsychiatric patients and those with $\mathrm{AD}[1,3$, $6,7]$. Evans found that performance on neuropsychological evaluations predicted functional capacity beyond negative symptoms in elderly with schizophrenia. However, these authors were unable to identify specific cognitive domains that impacted functional impairment. To date, most studies have not utilized comprehensive and conceptually sound measures to identify specific cognitive domains to predict particular areas of daily functioning [1].

The limited existing literature suggests a correlation between objective neuropsychological assessment and informant reported level of functioning. This relative dearth of literature may be attributable to the difficulty in accurately measuring everyday functioning [1]. Trained observer ratings of functional level may be the gold standard but are very time consuming and impractical in outpatient settings. Loewenstein et al. [8] found that family members' report of functional impairment is "extremely accurate" when compared with objective functional performance and is a useful mechanism to assess functioning [8].

The present study evaluated the link between specific neurocognitive measures and informant report of ADLs and IADLs in patients diagnosed with mild AD. As recommended by Beck et al., we address challenges in the current literature by utilizing a comprehensive neuropsychological battery to predict daily functioning in this clinical population.

To our knowledge no research has addressed the possibility of gender-related differences in the cognitive mechanisms required for select areas of daily functioning [9]. For instance, women inherently expend more effort than men in the area of dressing and grooming, which implies increased cognitive effort. The majority of studies have dealt with possible gender differences by covarying for gender, which tends to obscure any meaningful relationships that may be gender specific. Our study directly evaluates gender differences in the ability of specific neuropsychological tests and cognitive domains to predict functioning.

\section{Methods}

2.1. Sample. The total sample consisted of 202 (91 male, 111 female) participants who met criteria for $\mathrm{AD}$ enrolled in the Longitudinal Research Cohort of the Texas Alzheimer's Research Consortium (TARC). To reduce the effect of stage of decline on these measures, only individuals with a Clinical Dementia Rating scale global score of $\leq 1.0$ were included in the sample. Collateral information on ADLs and IADLs ratings was obtained from immediate caregivers who predominately were family members (spouse and or children) using the Lawton-Brody rating scales. The methodology of the TARC project has been described in detail elsewhere [10]. Briefly, the TARC project is a longitudinal multisite study of a cohort of $\mathrm{AD}$ patients and normal controls where each participant undergoes an annual evaluation that includes a medical examination, interview, neuropsychological testing, and blood draw. AD patients met consensus-based diagnosis for probable AD based on NINCDS-ADRDA criteria [11]. Male participants were 56 to 92 years of age $(M=74.36$, $\mathrm{SD}=8.21)$, and females participants were 54 to $92(M=$ $76.95, \mathrm{SD}=7.74)$. The characteristics of the participants are presented in Table 1 . The majority of participants were Caucasian (98\%), black or African American (1.5\%) was the next largest group. The TARC project received Institutional Review Board approval, and all participants and/or caregivers provided written informed consent.

2.2. Assessment. The TARC neuropsychological core battery consists of the following instruments: Wechsler Digit Span, Logical Memory, and Visual Reproduction, Trail Making Test A \& B, Clock Drawing Test (CDT), Boston Naming Test, the Geriatric Depression Scale (GDS-30), and the Clinical Rating scale (CDR). Verbal memory was assessed with the Wechsler Logical Memory I (LM I) and Wechsler Logical Memory II (LM II), visual memory was assessed with the Wechsler Visual Reproduction I (VRI) and II (VRII), attention was evaluated by performance on Trails A and Total Digit Span, linguistic capacity was assessed with Boston Naming Test (BNT) and verbal fluency (FAS, Category Naming (COWAT)), measures of executive functioning in this battery included the CDT and Trails B. Cognitive evaluation was administered in a controlled setting according to standardized instructions. In order to equate scores from digit span and story memory scales, all raw scores were converted to scale scores based on previously published normative data [12]. For the Boston Naming Test, the current group recently conducted an independent study that demonstrated the psychometric properties of an estimated 60-item BNT score that can be calculated from 30-item versions [13]. Adjusted scale scores were utilized as dependent variables in analyses. 
TABLE 2: $t$ values of significant predictors of IADLs in men and women.

\begin{tabular}{lcc}
\hline & LMI & Clock \\
\hline Total IADL & 2.34 & 3.78 \\
Medicine & - & 12.23 \\
Finance & 11.11 & - \\
Transport & - & 9.03 \\
Laundry & - & 7.67 \\
Housekeeping & - & 8.84 \\
Food prep & 3.86 & 2.94 \\
Shopping & 12.70 & - \\
Telephone & 12.63 & - \\
\hline
\end{tabular}

LM: logical memory, IADL: instrumental activities of daily living. All $P$ values $<.0001$.

2.3. Data Analysis. Descriptive statistics and one-way ANOVA comparison of male and female samples (presented in Table 1) were conducted using SPPS version 17.0. Stepwise regression modeling was used to evaluate the link between each test of cognitive function and ADLs and IADLs. Independent variables were caregiver ratings on the Physical Self-Maintenance Scale for ADLs and the Personal SelfMaintenance Scale for IADLs [14]. Each item has five descriptors from total independence to total dependence or total loss of functional control. The items are scored 0-4. The ADLs assessed were toileting, feeding, dressing, grooming, ambulation, and bathing. The IADLs assessed were telephone use, shopping, food preparation, housekeeping, transportation, laundry management of medications and finances. ApoE4 status (presence or absence) was also analyzed. Significance level was set at 0.05 .

\section{Results}

\subsection{Statistical Findings}

3.1.1. Predictors of IADL Functions for Total Sample. Logical Memory I (LMI) and performance on CDT were significant predictors of IADLs (see Table 2). Both LMI $(t=2.34$, $P=.022)$ and CDT $(t=3.78, P<.0001)$ significantly predicted Total IADL score (LMI/CDT combined; $t=14.38, P<$ .0001 ; Effect Size $\left(R^{2}=0.26\right)$. Evaluation of specific IADLs suggested that executive functioning (i.e., CDT) predicted independence with medication management $(t=12.23$, $\left.P<.0001 ; R^{2}=.32\right)$, transportation $(t=9.03, P<.0001$; $\left.R^{2}=.22\right)$, laundry $\left(t=7.67, P<.0001 ; R^{2}=.22\right)$, and housekeeping $\left(t=8.84, P<.0001 ; R^{2}=.30\right)$. Memory and learning capacity (LMI) significantly predicted ability to independently manage finances $\left(t=11.11, P<.0001 ; R^{2}=\right.$ $.24)$, shop $\left(t=12.70, P<.0001 ; R^{2}=.30\right)$, and telephone use $\left(t=12.63, P<.0001 ; R^{2}=.32\right)$. Measures of LMI and CDT $\left(t=11.36, P<.0001 ; R^{2}=.45\right)$ individually predicted food preparation ability. ApoE4 status was excluded from stepwise regression modeling and did not impact the level of IADL functioning in women and men with mild AD.
TABLE 3: $t$ values of significant predictors of ADLs in men and women.

\begin{tabular}{lcc}
\hline & Trails A & Clock \\
\hline Total ADL & - & 14.38 \\
Bathing & - & 10.34 \\
Grooming & - & 10.34 \\
Dressing & 11.18 & - \\
Feeding & - & 18.63 \\
\hline
\end{tabular}

ADL: activities of daily living.

All $P$ values $<.0001$.

TABLE 4: $t$ values of predictors of IADLs in women.

\begin{tabular}{lcccc}
\hline & LMI & LMII & BNT & Clock \\
\hline Total IADL & 3.73 & - & - & 2.15 \\
Medicine & - & - & - & 10.76 \\
Finance & 10.10 & - & - & - \\
Transport & - & - & 9.04 & - \\
Housekeeping & - & - & - & 8.35 \\
Food Prep & 3.86 & - & - & 2.04 \\
Shopping & 11.50 & - & - & - \\
Telephone & 3.80 & 2.43 & - & - \\
\hline
\end{tabular}

LM: logical memory, BNT: boston naming test; IADL: instrumental activities of daily living.

All $P$ values $<.0001$.

3.1.2. Predictors of ADL Functions of Total Sample. Performance on the CDT and Trails A significantly predicted ADL functions. Total ADL scores were predicted by score on the CDT $\left(t=14.38, P<.0001 ; R^{2}=.26\right)$. Likewise, CDT score predicted the ability to bath $\left(t=10.34, P<.0001 ; R^{2}=.24\right)$, groom $\left(t=10.34, P<.0001 ; R^{2}=.20\right)$, and feed/eat $\left(t=18.63, P<.0001 ; R^{2}=.28\right)$. Trails A predicted the patient's ability for self-dress $\left(t=11.18, P<.0001 ; R^{2}=.20\right)$ as demonstrated by Table 3. ApoE4 status was excluded from stepwise regression modeling and did not impact the level of ADL functioning in women and men with mild AD and as a result was not included in gender analyses.

\subsection{Gender Analyses}

3.2.1. Predictors of IADL Functions of Females. Significant predictors of the ability of female AD patients to perform IADLs included LMI, LMII, CDT, and the BNT. Total IADL score was predicted by performance on both LMI $(t=3.73$, $P<.0001)$ and CDT $(t=2.15, P=.035)$ with combined value of $\left(t=12.48, P<.0001 ; R^{2}=.49\right)$. The CDT alone significantly predicted independent functioning in the area of medication management $(t=10.76, P<.0001$; $\left.R^{2}=.38\right)$ and housekeeping $\left(t=8.35, P<.0001 ; R^{2}=\right.$ .36). Performance on LMI significantly predicted financial management $\left(t=10.10, P<.0001 ; R^{2}=.41\right)$ and the ability to shop independently $\left(t=11.50, P<.0001 ; R^{2}=.47\right)$. LMI and LMII significantly predicted telephone use $(t=11.25$, $P<.0001 ; R^{2}=.52$ ) and performance on the BNT alone predicted transportation or driving capacity $(t=9.04, P<$ $\left..0001 ; R^{2}=.25\right)$ in women with AD (see Table 4$)$. 
TABLE 5: $t$ values of predictors of ADLs in men.

\begin{tabular}{lccccc}
\hline & VRI & Trails A & BNT & Trails B & Clock \\
\hline Total ADL & 2.21 & 2.62 & - & - & - \\
Bathing & 6.26 & - & - & - & - \\
Grooming & 2.16 & - & - & 2.60 & - \\
Feeding & - & - & 2.94 & - & 2.42 \\
\hline
\end{tabular}

VR: visual reproduction; BNT: boston naming test; ADL: activities of daily living.

All $P$ Values $<.0001$.

3.2.2. Predictors of ADL Functions of Females. Levels of ADL functioning in women was predicted by performance on the CDT and Trails A. Performance on CDT predicted total ADL score $\left(t=11.55, P<.0001 ; R^{2}=.27\right)$ and bathing capacity $\left(t=8.37, P<.0001 ; R^{2}=.26\right)$. Ability to eat $/$ feed $(t=$ $\left.14.72, P<.0001 ; R^{2}=.32\right)$ independently was predicted by performance on Trails A.

3.2.3. Predictors of IADL Functions of Males. Performance on measures of VRI $(t=2.74, P=.010)$, VRII $(t=2.29$, $P=.029)$, Trails B $(t=2.74, P=.010)$, and COWAT $(t=$ 2.56, $P=.015)$ predicted medication management capacity in men $\left(t=4.67, P<.0001 ; R^{2}=.27\right)$. Food preparation was predicted by performance on the BNT $(t=5.18, P<.0001$; $\left.R^{2}=.38\right)$.

3.2.4. Predictors of ADL Functions of Males. VRI, Trails A, BNT, Trails B, and CDT were all significant predictors of level of ADL functioning in men with AD (see Table 5). Total ADL score $\left(t=8.86, P<.0001 ; R^{2}=.35\right)$ was significantly predicted by VRI and Trails A. Performance on VRI predicted bathing ability $\left(t=6.26, P<.0001 ; R^{2}=.41\right)$ and when combined with Trails $B$ also predicted grooming capacity $\left(t=5.66, P<.0001 ; R^{2}=.51\right)$. The ability to eat/feed independently was predicted by BNT and CDT $(t=16.65$, $\left.P<.0001 ; R^{2}=.56\right)$.

\section{Discussion}

Previous research has shown that cognitive functioning, as assessed by neuropsychological tests, is the strongest predictor of functional impairment $[6,7]$. Specific cognitive domains of executive functioning, praxis/visuospatial skills, and memory have been found to be useful for predicting ADL and IADL in assisted-living elders [6]. Measures of executive functions have been shown to predict IADLs in community dwelling elders [15]. Our findings are consistent with previous research and demonstrate a significant relationship between performance of daily living activities and neurocognitive performance. Unlike other studies, we found that attention is an important predictor of ADLs in $\mathrm{AD}$ patients. These differences may be related to differences in setting. Prior studies have been conducted in assisted care facilities where caregiver assistance may be sufficient to overcome inattention. However, individuals with mild dementia seen as outpatients in our study were likely responsible for

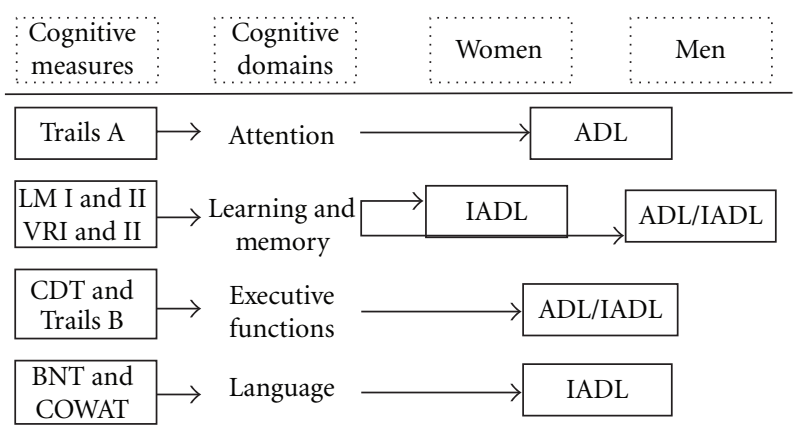

FIgure 1: Predicting ADL and IADLs in men and women with AD.

basic ADLs, and, thereby, attention was necessary to facilitate functioning.

Memory and learning (LMI, LMII, VR I, VRII), executive functioning (CDT, Trails B), and language (BNT, COWAT) were significant predictors of ADLs and IADLs. Among the measures administered, CDT, LMI, and Trails A were predictive of both ADL and IADL functioning in analysis of the total sample. Whereas prior reports suggested that cognitive abilities are most predictive of complex tasks of everyday functioning $[15,16]$, our results suggest that cognitive test performance also predicts basic ADLs (e.g., bathing, grooming, dressing, and feeding). This suggests that even in patients with mild $\mathrm{AD}$, basic ADLs likely also require complex cognitive processes.

Another intriguing finding is that the presence of ApoE4 genotype was not predictive of level of IADL and ADL functioning in the current sample. Presence of particular APOE genotype has been associated with greater disability in prior research with patients with MCI [17]. The current data suggests that the presence of APOE was not significantly associated with level of functioning in patients who have converted to AD status. This is the first known study to directly examine gender differences. We anticipated gender differences because there are (a) differences in task performance and (b) differences in strategies used to perform ADLs and IADLs. For instance, it has been documented that women tend to use landmarks when driving and given directions where men are more likely to use street names $[9,18]$. In our research we administered several measures within each cognitive domain to facilitate understanding of not only which domain is different for male and female but also which specific measure best predicts functioning.

Figure 1 demonstrates gender differences in ADLs and IADLs with regard to specific cognitive domains assessed. Measures of attention predicted overall ADL scores in both men and women. However, attention predicted bathing and eating ability in women but not men. Language also predicted IADL functions in men (food preparation) and women (driving). Executive function predicted both ADLs and IADLs in women and men. Gender differences remained in the domain of learning and memory, suggesting that men rely on this process for both ADL and IADL whereas it is only predictive of IADL functions in women. 
A notable gender difference is that cognitive functioning is generally a better predictor of ADL and IADL functioning for women compared to men. While the administered assessment battery predicted practically all daily tasks for women, it only predicted a few specific ones for men. This may be due in part to the likelihood that men especially of the generation in our sample are less likely to be involved in cooking, shopping, housekeeping and laundry and hence have little variability. For men, the IADL of medicine management was the only area predicted by performance on several different cognitive measures. Among ADLs, only bathing, grooming, and feeding capacity was significantly predicted by VRI, Trails A and Trails B, BNT, and the CDT. However, in women, the CDT, verbal learning and memory (LMI and LMII) and language were good predictors of capacity to perform almost all ADLs and IADLs. These findings suggest that men tended to depend on visual learning and visual memory and women on verbal learning and verbal memory. LMI and LMII were predictive of functioning for women, whereas VRI and VRII were predictive for men. One could speculate that women tend to problem-solve verbally using "self-talk" whereas men tend to conceptualize visually.

The CDT appears to be a good measure in predicting functioning for women, but not for men. The CDT is typically seen as a measure of executive functioning and of frontal lobe processes. The clinical utility of the Clock Drawing Test has been documented [18] for diagnosing patients with dementia [19], but its relationship to specific functional activities has not been reported.

The generalizability of our findings suffers from the relatively small sample size and the nature of subject recruitment. The current study is one of the first to examine gender differences, and efforts to replicate these findings is warranted due to several sample limitations that include lack of racial diversity and differences in educational levels and age among men and women in this sample. Though it is unlikely these factors negate current results, it would be best to stratify according to education and age in future studies. Additional studies with larger more representative samples are needed to further assess the impact of gender on predicting functioning. Although late-life depression could impact cognitive functioning, gender differences were not significant (Table 1) in our sample, and, therefore, depression score was not accounted for in the analyses. However, effects of depression are significant and warrant future efforts.

Although the current research has its limitations this study has several advantages over earlier studies in terms of understanding patients with AD. First, the sample was limited to individuals with mild AD which helps control for the affect of disease severity on functional activities. Second, it examines gender differences that have not been examined in prior research. Third, in addition to evaluating predictive value of specific domains it also evaluated specific measures within those domains in both gender-specific and mixedgender analyses. The findings of this study underscore the importance of gender and the gender-specific relations of neurocognitive measures to everyday activities.

\section{Conclusion}

Acknowledging gender differences is important as it may facilitate more accurate interpretation of neurocognitive tasks and its relationship to particular daily living activities. These findings also have clinical values for making informed decisions and recommendations of capacity in patients with $\mathrm{AD}$. There is generally consensus that executive functioning is an important predictor of capacity to perform complex tasks (IADLs; 15). While this may be accurate for women, current findings suggest that it may be an irrelevant predictor for men. Understanding how to most accurately predict level of function will also enable patients to maintain daily functions longer, reducing caregiver fatigue, and also social and economic burden.

\section{Acknowledgments}

This paper was made possible by the TARC, funded by the state of Texas through the Texas Council on Alzheimer's Disease and Related Disorders. No authors expressed any conflicts of interest with this project.

\section{References}

[1] C. K. Beck and L. B. Frank, "Assessing functioning and selfcare abilities in Alzheimer disease research," Alzheimer Disease \& Associated Disorders, vol. 11, no. 6, pp. 73-80, 1997.

[2] A. Busse, J. Bischkopf, S. G. Riedel-Heller, and M. C. Angermeyer, "Subclassifications for mild cognitive impairment: prevalence and predictive validity," Psychological Medicine, vol. 33, no. 6, pp. 1029-1038, 2003.

[3] S. Tekin, L. A. Fairbanks, S. O'Connor, S. Rosenberg, and J. L. Cummings, "Activities of daily living in Alzheimer's disease: neuropsychiatric, cognitive, and medical illness influences," The American Journal of Geriatric Psychiatry, vol. 9, no. 1, pp. 81-86, 2001.

[4] S. Gauthier, I. Gelinas, and L. Gauthier, "Functional disability in Alzheimer's disease," International Psychogeriatrics, vol. 9, no. 1, pp. 163-165, 1997.

[5] R. L. Ernst and J. W. Hay, "The US economic and social costs of Alzheimer's disease revisited," American Journal of Public Health, vol. 84, no. 8, pp. 1261-1264, 1994.

[6] D. J. Burdick, A. Rosenblatt, Q. M. Samus et al., "Predictors of functional impairment in residents of assisted-living facilities: the Maryland assisted living study," The Journals of Gerontology, vol. 60, no. 2, pp. 258-264, 2005.

[7] J. D. Evans, R. K. Heaton, J. S. Paulsen, B. W. Palmer, T. Patterson, and D. V. Jeste, "The relationship of neuropsychological abilities to specific domains of functional capacity in older schizophrenia patients," Biological Psychiatry, vol. 53, no. 5, pp. 422-430, 2003.

[8] D. A. Loewenstein, S. Arguelles, M. Bravo et al., "Caregivers' judgments of the functional abilities of the Alzheimer's disease patient: a comparison of proxy reports and objective measures," The Journals of Gerontology, vol. 56, no. 2, pp. P78P84, 2001.

[9] L. A. Cushman and C. J. Duffy, "The sex specificity of navigational strategies in Alzheimer disease," Alzheimer Disease and Associated Disorders, vol. 21, no. 2, pp. 122-129, 2007.

[10] S. Waring, S. E. O’Bryant, J. S. Reisch, R. Diaz-Arrastia, J. Knebl, and R. Doody, "The Texas Alzheimer's research 
consortium longitudinal research cohort: study design and baseline characteristics," Texas Public Health Journal , vol. 60, no. 3, pp. 9-13, 2008.

[11] G. McKhann, D. Drachman, M. Folstein, R. Katzman, D. Price, and E. M. Stadlan, "Clinical diagnosis of Alzheimer's disease: report of the NINCDS-ADRDA work group under the auspices of department of health and human services task force on Alzheimer's disease," Neurology, vol. 34, no. 7, pp. 939-944, 1984.

[12] B. A. Steinberg, L. A. Bieliauskas, G. E. Smith, and R. J. Ivnik, "Mayo's older Americans Normative studies: age- and IQadjusted norms for the Trail-Making Test, the Stroop Test, and MAE controlled Oral Word Association Test," Clinical Neuropsychologist, vol. 19, no. 3-4, pp. 329-377, 2005.

[13] V. L. Hobson, J. R. Hall, M. Harvey et al., "An examination of the Boston Naming Test: calculation of "estimated" 60-item score from 30- and 15-item scores in a cognitively impaired population," International Journal of Geriatric Psychiatry, vol. 26, no. 4, pp. 351-355, 2011.

[14] M. P. Lawton and E. M. Brody, "Assessment of older people: self-maintaining and instrumental activities of daily living," Gerontologist, vol. 9, no. 3, pp. 179-186, 1969.

[15] D. A. Cahn-Weiner, P. A. Boyle, and P. F. Malloy, "Tests of executive function predict instrumental activities of daily living in community-dwelling older individuals," Applied Neuropsychology, vol. 9, no. 3, pp. 187-191, 2002.

[16] M. McCue, J. C. Rogers, and G. Goldstein, "Relationships between neuropsychological and functional assessment in elderly neuropsychiatric patients," Rehabilitation Psychology, vol. 35, no. 2, pp. 91-99, 1990.

[17] A. Kulminski, S. V. Ukraintseva, K. G. Arbeev et al., "Association between APOE $\varepsilon 2 / \varepsilon 3 / \varepsilon 4$ polymorphism and disability severity in a national long-term care survey sample," Age and Ageing, vol. 37, no. 3, pp. 288-293, 2008.

[18] K. I. Shulman, "Clock-drawing: is it the ideal cognitive screening test?" International Journal of Geriatric Psychiatry, vol. 15, no. 6, pp. 548-561, 2000.

[19] A. Wiechmann, J. R. Hall, and S. E. O'Bryant, "The utility of the spatial span in a clinical geriatric population," Aging, Neuropsychology, and Cognition, vol. 18, no. 1, pp. 56-63, 2010. 


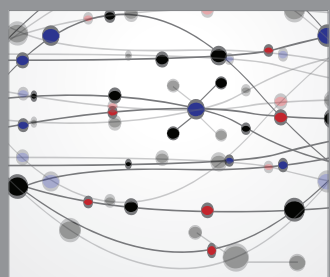

The Scientific World Journal
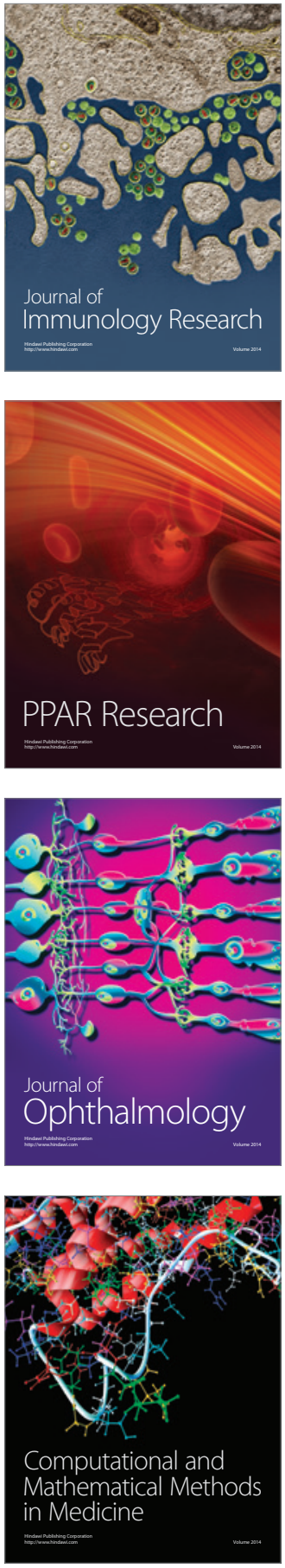

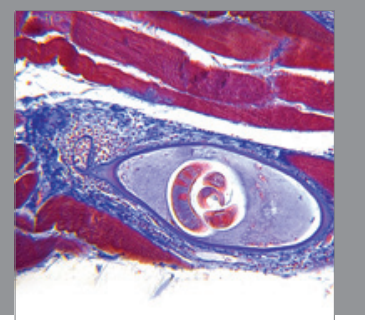

Gastroenterology

Research and Practice
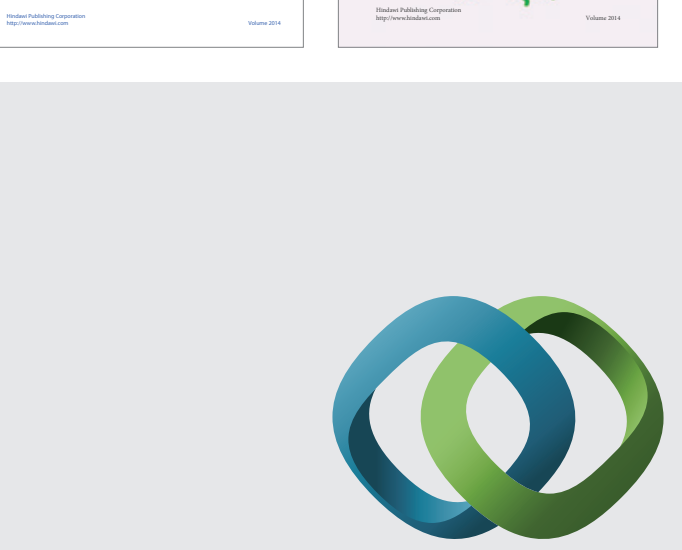

\section{Hindawi}

Submit your manuscripts at

http://www.hindawi.com
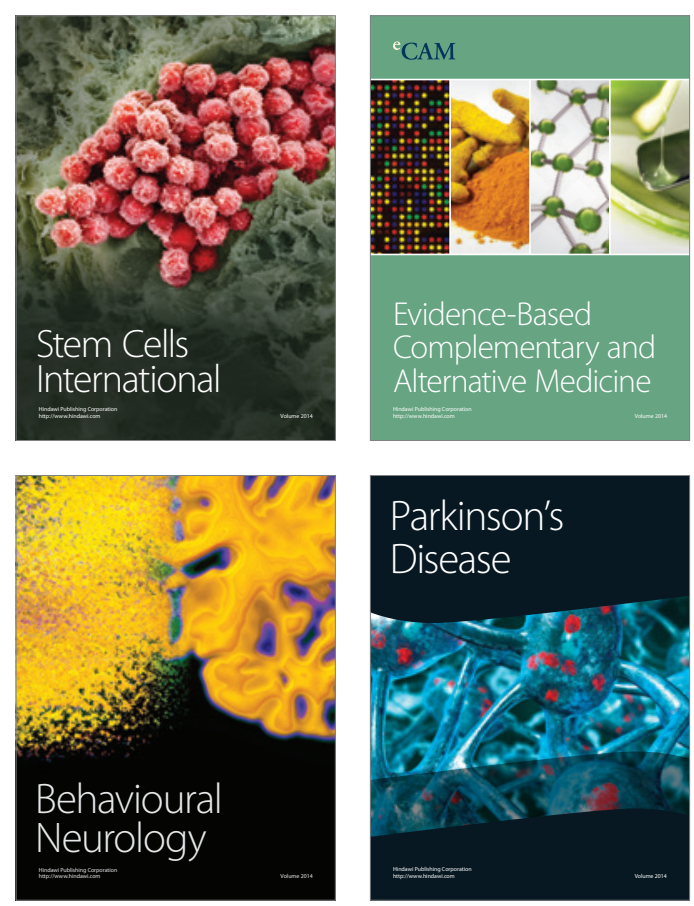

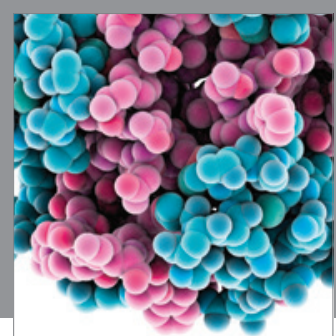

Journal of
Diabetes Research

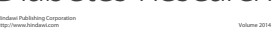

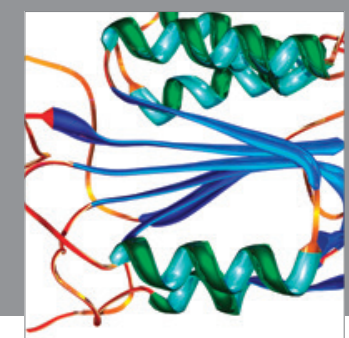

Disease Markers
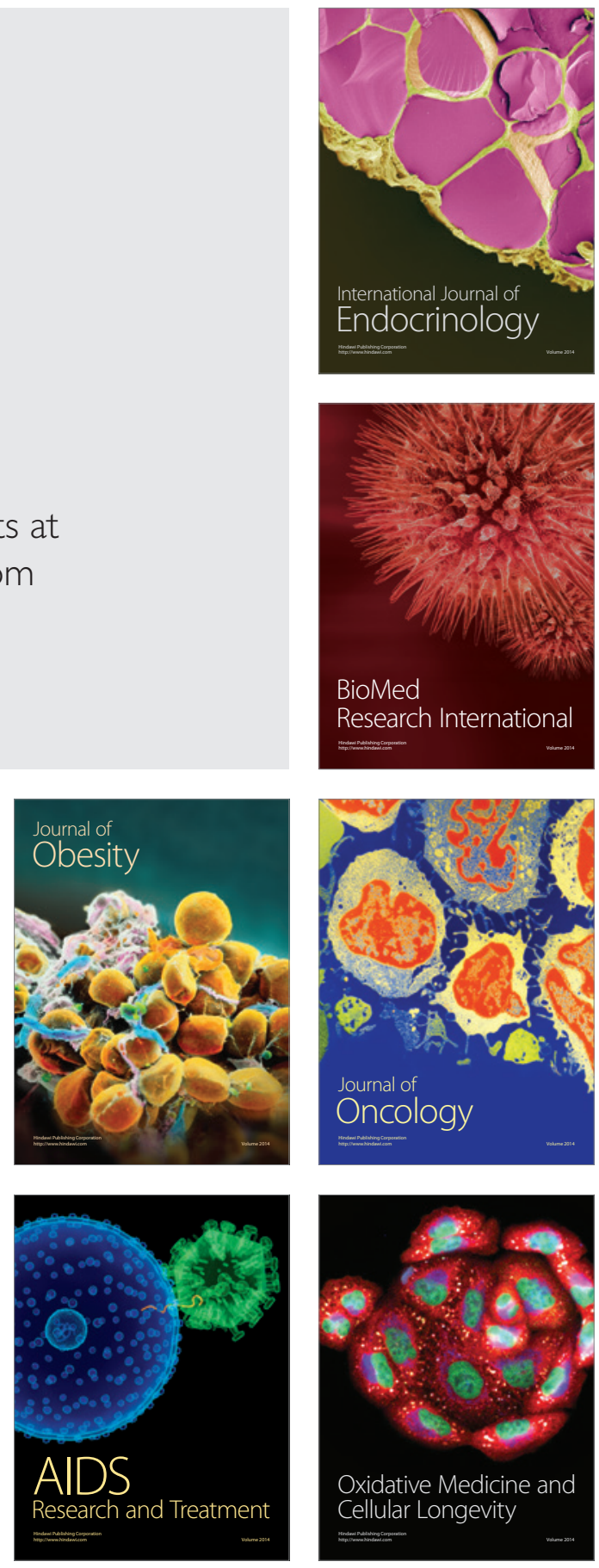